Revue

d'ethnoécologie
Revue d'ethnoécologie

6 | 2014

Conservation de la nature : quel rôle pour les sciences sociales?

\title{
Protéger les humains et les non-humains
}

L'exemple des Bunaq de Lamaknen

Protect humans and non-humans: the example of the Bunaq from Lamaknen

\section{Claudine Friedberg}

\section{(2) OpenEdition}

Journals

Édition électronique

URL : http://journals.openedition.org/ethnoecologie/1875

DOI : $10.4000 /$ ethnoecologie. 1875

ISSN : 2267-2419

Éditeur

Laboratoire Eco-anthropologie et Ethnobiologie

Référence électronique

Claudine Friedberg, "Protéger les humains et les non-humains », Revue d'ethnoécologie [En ligne],

6 | 2014, mis en ligne le 31 décembre 2014, consulté le 19 avril 2019. URL : http://

journals.openedition.org/ethnoecologie/1875 ; DOI : 10.4000/ethnoecologie.1875

Ce document a été généré automatiquement le 19 avril 2019.

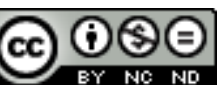

Revue d'ethnoécologie est mis à disposition selon les termes de la licence Creative Commons Attribution - Pas d'Utilisation Commerciale - Pas de Modification 4.0 International. 


\title{
Protéger les humains et les non- humains
}

\author{
L'exemple des Bunaq de Lamaknen ${ }^{1}$ \\ Protect humans and non-humans: the example of the Bunaq from Lamaknen
}

\section{Claudine Friedberg}

1 Les politiques de conservation de la biodiversité in situ ne peuvent se développer sans la participation des populations locales.

2 Il importe donc de se demander à quoi correspond dans leur conception du fonctionnement du monde et leur relation à celui-ci, ce que les scientifiques désignent par le terme biodiversité. Quels niveaux de cette dernière sont concernés par leurs pratiques: celui des écosystèmes, des espèces, des variétés et races des plantes et animaux domestiqués? Il importe surtout de comprendre quelles peuvent être leurs motivations et leurs pratiques qui aboutissent, ou pas, à conserver certains éléments de leur environnement.

3 En effet les pratiques ayant pour résultat la conservation de certains éléments de l'environnement d'une population peuvent avoir des motivations multiples, souvent éloignées de celles des organismes scientifiques internationaux de conservation de la nature.

4 En me concentrant uniquement sur le monde végétal je me propose de décrire les pratiques que j'ai pu observer dans les années 1970 chez une population du centre de Timor dans la partie indonésienne de l'île, à Abis². Ce village, dont les habitants sont de langue bunaq, est situé dans la partie haute du district de Lamaknen dont le territoire était alors frontalier avec la partie portugaise de l'île, aujourd'hui Timor Leste (Figures 1, 2 et 3). Le bunaq est une langue non austronésienne qui a cependant emprunté un certain nombre de caractéristiques dans le vocabulaire et la syntaxe aux langues austronésiennes qui l'entourent (Schapper 2011). 
Figure 1 : Carte de Timor

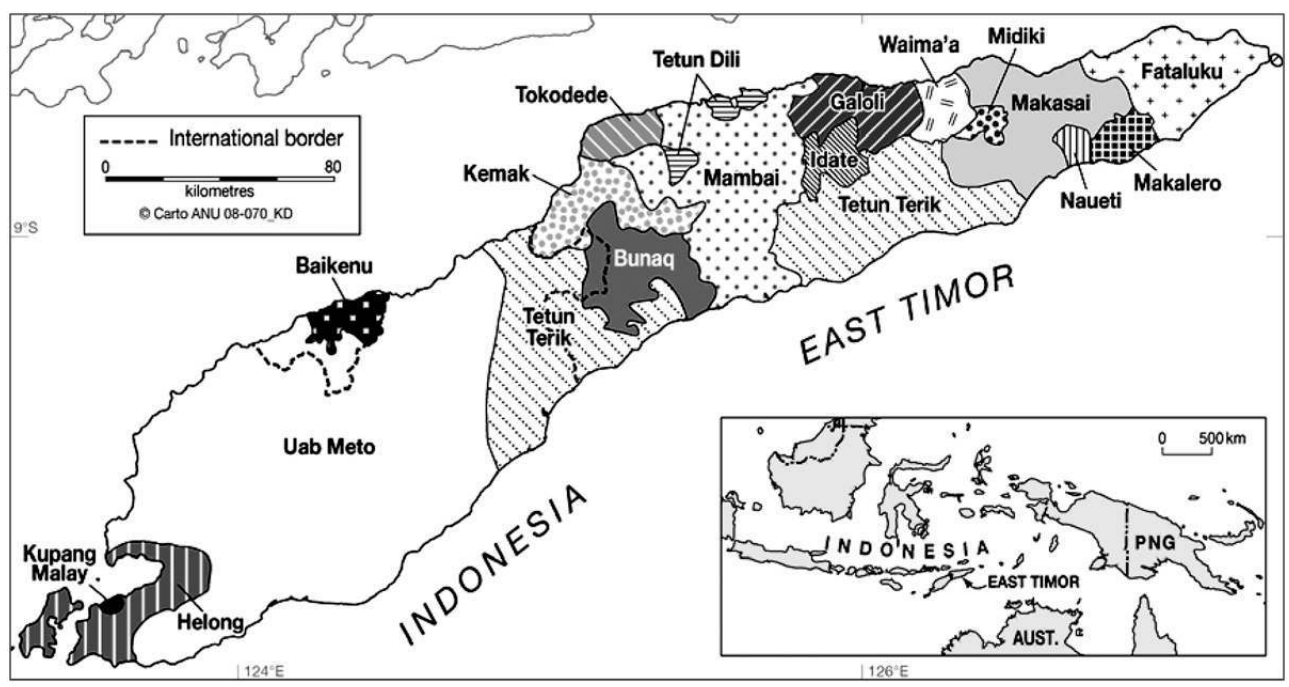

Tirée de Schapper (2011 : 30)

Figure 2 : Carte du territoire de Lamaknen

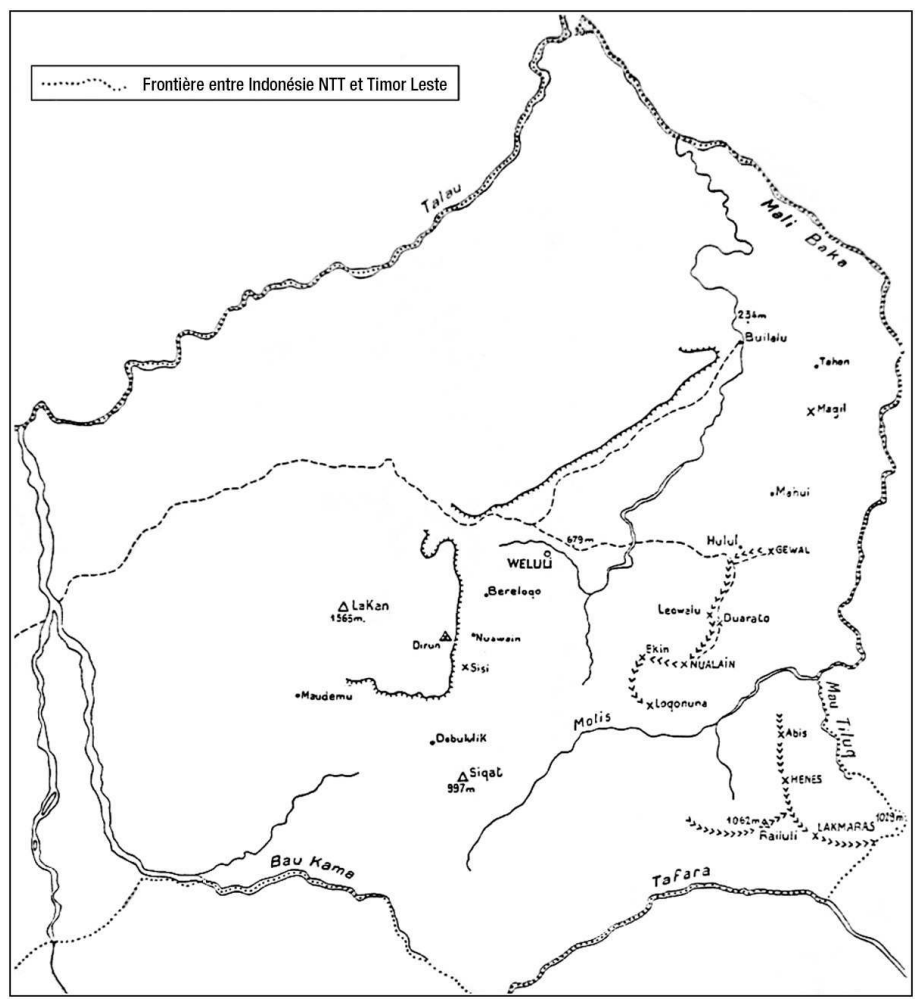

Tirée de Friedberg (1982 : 1636) 
Figure 3 : Carte du territoire d'Abis

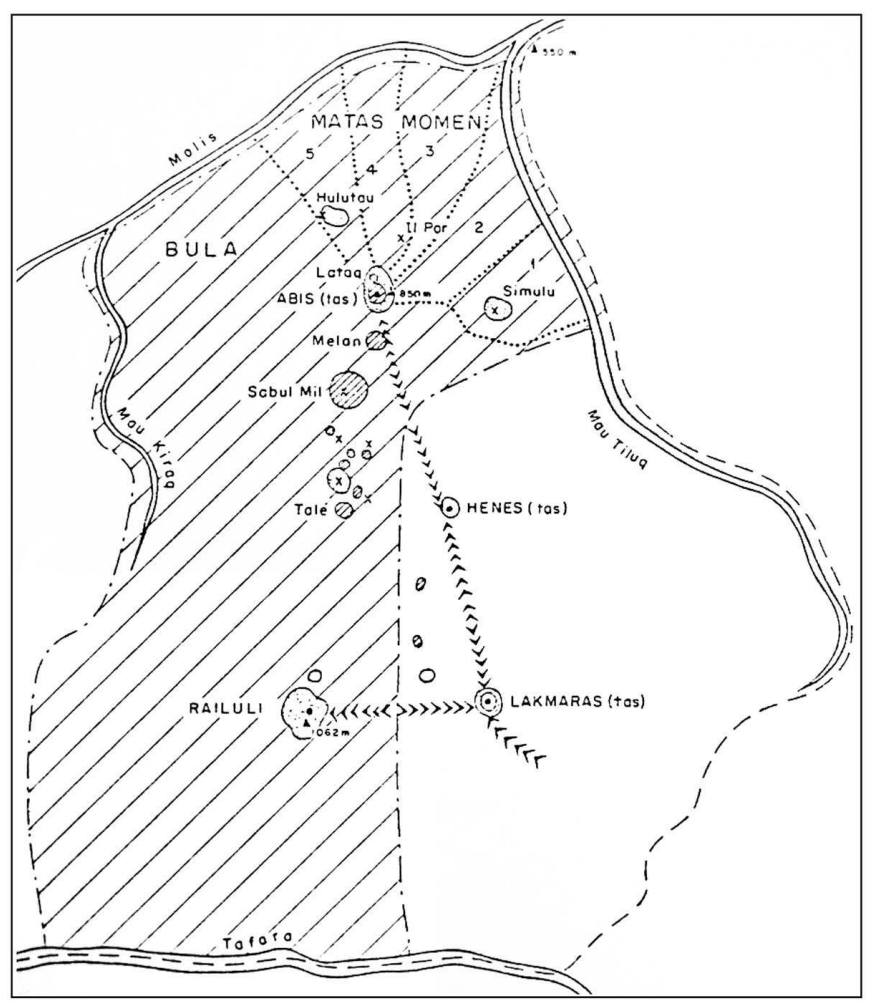

Tirée de Friedberg (1982: 1637)

5 Les habitants de ce village considèrent que leurs ancêtres sont des immigrants sur leur territoire dont ils ont chassé les occupants précédents appelés Melus, une dénomination qui ne correspond à aucune population connue, mais utilisée dans la région pour désigner les autochtones. Pour ces villageois « les maître du sol » de leur territoire sont les esprits de ces Melus.

6 Cependant, même s'ils estiment qu'il n'y a aucun descendant de ces Melus dans le village, une des maisons s'est vue attribuer les fonctions liées à la gestion de son territoire et à la responsabilité vis-à-vis des autres êtres qui y vivent, en particulier des végétaux, en tant qu'héritière de la maison de l'ancien chef féminin.

7 Selon le récit des villageois sur la façon dont leurs ancêtres ont occupé le village, ceux qui l'occupaient ont fui ou ont été tués. Toutefois dix seraient revenus, trop âgés pour avoir des enfants, et parmi eux l'ancien chef féminin. Il devint alors le chef rituel de sa maison, investie par certains des nouveaux arrivants, qui hérite de toutes les fonctions liées à la protection et la gestion des ressources du territoire tandis qu'une nouvelle maison détient la fonction de dato pana « chef féminin ».

8 La maison est chez les Bunaq l'unité de base de la société. À Abis il y en avait vingt quand j'ai mené mon enquête. Le pouvoir politique y était dyarchique comme dans toute la région avec au sommet un chef féminin et un chef masculin, le premier ayant un statut supérieur au second, les deux fonctions étant l'une comme l'autre détenues par des hommes ${ }^{3}$. 


\section{Les pratiques de conservations liées à l'agriculture}

9 L'ensemble du territoire du village est partagé en deux parties. L'une, appelée matas momen, est consacrée à la culture et interdite à tous les animaux domestiques dont les excréments ne peuvent donc servir d'engrais. Elle est entourée d'une clôture collective, consistant en un petit muret de pierres. L'autre, appelée bula, comporte de la prairie (c'est le sens du terme bula) et des zones arborées et arbustives plus ou moins étendues. C'est un espace où les animaux domestiques (porcs, buffles, bovins, chèvres et chevaux) peuvent se déplacer librement. Quand des individus font des champs sur bula ils doivent pour les protéger construire une haie autour, haies vives ou barrières faites de branchages.

10 Les Bunaq pratiquent une agriculture sur brûlis. À Abis l'ensemble du matas momen est partagé entre les différentes maisons. Une expression désignant cet ensemble, soron gontiet «les cinq soles» et différents détails du cycle de trois ans des rituels accompagnant la mise en culture, tendent à indiquer qu'au départ on ouvrait alternativement un champ dans l'une des cinq soles utilisée trois années de suite. On revenait donc exploiter la même sole au bout de 15 ans durant lesquels la végétation pouvait se régénérer. Ce système ne pouvait fonctionner que si chaque maison avait un droit d'usage sur une portion de chacune des cinq soles.

11 Lorsque j'ai mené mes recherches dans les années 1970 ce système d'assolement ne fonctionnait plus, sans doute parce qu'Abis avait accueilli plusieurs nouvelles maisons auxquelles on avait donné des droits d'usage regroupés sur des espaces d'un seul tenant. Le rythme d'utilisation d'une même parcelle était souvent inférieur à 15 ans, ce qui rendait d'autant plus nécessaire la gestion de sa végétation lors des brûlis de préparation des champs. Tout d'abord toutes les espèces utiles comme les manguiers, les palmiers à sucre ou le santal ${ }^{4}$ étaient protégées en les entourant avec des feuilles de bananier. Ensuite on se contentait d'élaguer les arbres dont on voulait non seulement qu'ils repoussent lors de l'abandon du champ, mais aussi qu'ils se reproduisent (Photographie $1^{5}$ ). C'était le cas de plusieurs espèces autochtones comme Melochia umbellata ${ }^{6}$ ou Cassia timoriensis, mais aussi et surtout du Sesbania grandiflora et Leucaena leucocephala (Fabacées) introduites par les Néerlandais.

Même en dehors des périodes de culture, le matas momen était donc recouvert d'une végétation qui restait sous la dépendance des humains. 


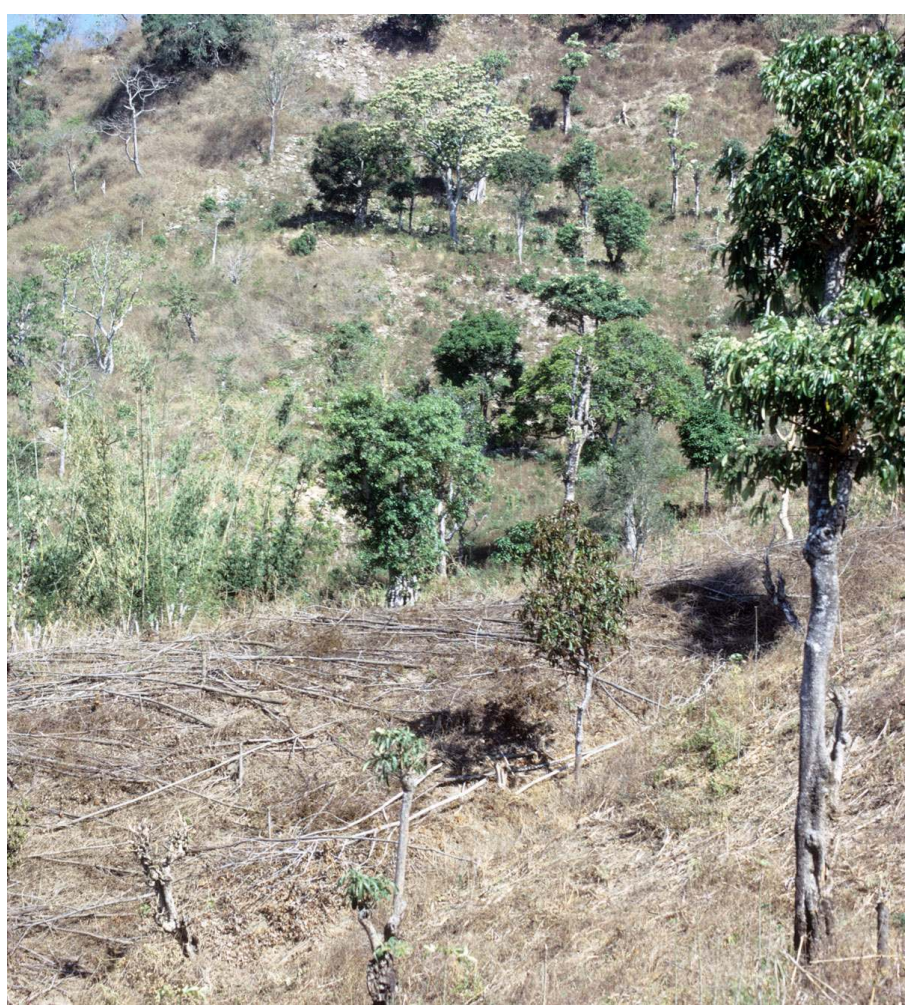

Au premier plan des troncs d'arbres coupés qui sèchent avant d'être brûlés et, ça et là, des arbres plus âgés qui ont été préservés, sans doute déjà lors d'essartages précédents, comme le manguier à droite (c) C. Friedberg

13 Au moment des brûlis de préparation des champs, des précautions étaient prises, afin que le feu ne puisse se propager en dehors de la parcelle qui sera cultivée. Sur les espaces brûlés non cultivés se propage en effet Imperata cylindrica, une Poacée à stolon très difficile à éradiquer ensuite, et qui empêche d'autres espèces de pousser. Toutefois les Bunaq s'efforçaient d'en conserver sur certaines parties limitées de leur territoire car cette herbe était indispensable pour couvrir la toiture de leur habitation.

Il faut ajouter que, dans les conditions de relief très accidenté qui règnent au centre de Timor, il est indispensable de préserver une couverture végétale, tout espace dénudé étant susceptible d'être attaqué par l'érosion durant la saison des pluies.

\section{La biodiversité des espèces cultivées}

La majeure partie des champs était consacrée au maïs, céréale introduite par les Portugais dès le $\mathrm{XVII}^{\mathrm{e}}$ siècle et qui constituait la nourriture de base. Seules quelques parcelles dont le sol était considéré comme propice, étaient utilisées pour cultiver du riz, généralement de façon collective par tous les membres d'une maison quand une fête concernant cette dernière (reconstruction, réfection du toit ou cérémonie funéraire) était programmée. Le riz était en effet resté la nourriture de prestige, obligatoire lors des rituels pour les offrandes aux êtres de l'au-delà, et pour offrir aux hôtes.

16 Ce riz de montagne est cultivé à sec; il n'existait, à l'époque, que quelques parcelles inondables à Lamaknen, mais aucune à Abis. Les habitants de ce village distinguaient 
plusieurs variétés de riz qu'ils cultivaient ensemble dans le même champ; certaines avaient été introduites récemment mais ils considéraient qu'elles s'étaient modifiées sous l'influence des variétés anciennes et réciproquement; il s'agissait évidemment de «variétés populations ".

Les variétés de maïs étaient distinguées en fonction de leur durée de maturation, très précoces, moins précoces, tardives ; puis à l'intérieur de ces trois variétés des distinctions étaient faites selon la couleur des épis.

D'autres plantes alimentaires étaient cultivées en mélange dans les champs de maïs comme divers "haricots" (Vigna sp., Cajanus cajan, Lablab sp., Phaseolus sp.) et des courges, mais peu de tubercules autochtones : quelques taros (Colocasia sp. et Xanthosoma sp.) et surtout des ignames (Dioscorea alata dont 14 variétés étaient distinguées d'après leur forme, la couleur de leur peau et celle de leur chair ; 11 variétés de Dioscorea esculenta étaient distinguées de la même façon). Quelques patates douces étaient repiquées après la récolte du maïs pour être consommées durant la période de soudure. Généralement les tubercules étaient consommés au champ quand on y allait pour effectuer différents travaux et rarement rapportés à la maison.

On cultivait aussi quelques céréales archaïques comme le millet (Setaria italica) et le Coix lacryma jobi. Je n'ai vu utiliser les graines de ce dernier que pour la confection de gâteaux déposés sur les tombes à l'occasion des rituels précédant les semailles.

Cependant déjà dans les années 1970 la culture du manioc se développait comme réserve alimentaire sur pied en cas de mauvaise récolte de maïs, très sensible aux aléas climatiques, en raison de l'irrégularité des pluies de mousson et de la mauvaise fécondation due à des vents forts soufflant en période de pollinisation.

Il faut aussi préciser qu'à l'époque, les habitations installées sur bula étaient entourées d'un jardin dans lequel on cultivait des papayers, quelques pieds de canne à sucre et des bananiers. Pour ces deux dernières plantes, on se trouve à Timor à proximité de la zone d'origine de leur domestication. On peut donc penser y trouver des variétés anciennes, mais il faudrait une étude génétique que je n'ai pas faite. Néanmoins j'ai recueilli à Lamaknen des termes pour quatre clones de canne à sucre et dix-sept pour les bananiers. Parmi ces derniers on peut repérer des variétés introduites du reste de l'Indonésie mais l'une d'elles, comportant cinq sous-variétés, est considérée par les informateurs comme très ancienne, car elle figure dans les mythes et est obligatoirement utilisée dans les rituels de naissance.

En dehors des plantes alimentaires signalons la culture du bétel (Piper betle) sur des tuteurs près des habitations et de l'aréquier près des points d'eau. Leur consommation, des feuilles pour celui-là, de l'intérieur du fruit pour celui-ci, était quotidienne.

Même s'ils se reproduisaient seuls, les bambous dont les usages sont multiples sont considérés par les Bunaq comme ayant été transportés par leurs ancêtres au cours de leurs migrations. Il en existe quatre espèces: Dendrocalamus asper, Bambusa vulgaris, Schizostachium brachycladum et S. blumii. Seul un bambou épineux (Bambusa blumeana) serait autochtone.

Pour plusieurs espèces utiles il est difficile de dire si elles étaient cultivées ou simplement protégées ; c'est le cas du bancoulier dont la noix était jadis la seule source d'huile pour l'éclairage ou des pandanus utilisés pour fabriquer des nattes et des paniers. Par contre il $\mathrm{y}$ avait pour le palmier à sucre un interdit de plantation. 

débuts de l'agriculture à Timor se seraient effectués dans des agroforêts (Donohue \& Denham 2010, Oliveira 2008, O'Connor 2007).

\section{L'habitat, les relations entre les différents êtres, les racines des humains et les âmes des plantes}

habitants d'Abis résidaient dans le village traditionnel ou tas situé au sommet d'une
colline. Tous les membres d'une maison logeaient dans la même habitation disposée autour de la place de réunion (mot) et du lieu de culte collectif situé sur le bord de celleci, le bosok.

Cet autel consiste en un cylindre composé de pierres entassées qui est censé être la matérialisation de la vitalité des membres du village, désignée par l'expression pana getel mone goron " racines des femmes et feuilles des hommes ». Comme souvent dans la région, on utilise des métaphores végétales pour parler de ce qui concerne la vie des humains. Dans les généalogies mythiques, les épouses sont appelées biso bin « semences ». Quand elles quittent leur maison d'origine, on dit sasa o sulol pili o paqe « rejet et bouture cueillir et détacher ", c'est-à-dire que l'on les considère comme des pousses « repiquées » dans la maison de leur époux.

Cette proximité entre le monde végétal et celui des humains se manifeste également par le fait que l'on attribue des âmes aux plantes comme aux humains. Ainsi, avant la récolte du riz est-il nécessaire d'accomplir un rituel pour appeler son âme, sinon, dit-on, les épis seront vides (Photographie 2).

Photographie 2 : Récolte du riz

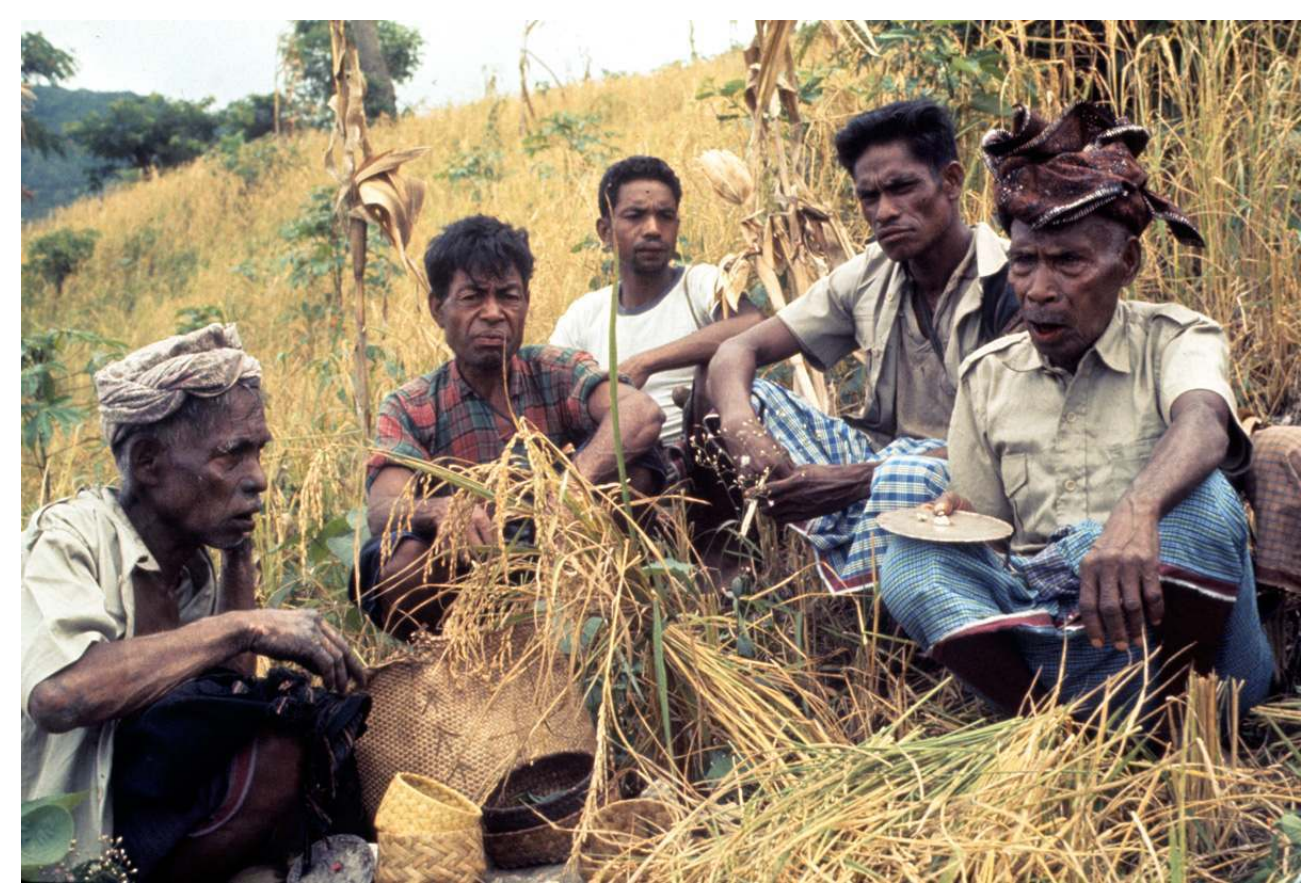

Avant de commencer à couper le riz un maître de la parole récite un texte pour appeler son âme (c) C. Friedberg

Revue d'ethnoécologie, 6 | 2014 
Qund on coupe des plantes, et en particulier des arbres pour construire des habitations, il faut également prendre des précautions rituelles. Une certaine ambiguïté demeure pourtant quant à l'objet des rituels que l'on accomplit : s'adressent-ils à la plante ellemême ou à ses "parents »? Dans les récits mythiques tels que j'ai pu les recueillir en 1969/1970, dès les débuts du monde, parmi les êtres créés dans le ciel par un couple primordial, il y a la mère et le père du bétel et de la noix d'arec, mais aussi la mère de tous les arbres qui constituent les maisons et celle de tous les autres végétaux servant dans leur construction. Ces êtres mythiques apparaissent en même temps que ceux qui donneront naissance aux humains.

C'est lors de l'abattage d'un arbre que le sens du rituel apparaît le plus clairement. Je ne l'ai vu accomplir qu'une seule fois lors de la coupe d'un arbre pour fabriquer un cercueil. Une chèvre a été sacrifiée pour hotel gawa gemen, "redresser l'extrémité de l'arbre ", c'est-à-dire pour renvoyer vers le monde d'En haut l'âme de l'arbre censée être transportée sur les cornes de la chèvre. Des offrandes comportant du bétel et de la nourriture confectionnée avec la chair de la chèvre et du riz sont considérées comme " une compensation pour la mère des arbres dont on a tué un enfant ».

31 Le même type de cérémonie devait se faire théoriquement lors de l'essartage d'un champ mais je ne l'ai jamais vu faire, sans doute parce qu'il n'y avait plus de gros arbres sur les champs que l'on réutilisait trop vite après l'abandon précédent. Cependant on m'a dit que si un homme qui réussissait ses cultures tombait malade de façon inexplicable, on supposait que c'était les arbres qu'il avait abattus qui étaient en colère contre lui. Il fallait alors effectuer un rituel destiné à séparer son âme de celles des arbres qu'il avait tués, rituel analogue à celui que l'on exécutait en cas du meurtre d'un humain par un autre afin de séparer l'âme de l'assassin de celle du mort.

On m'a aussi raconté comment, lorsqu'il s'agissait de couper un grand arbre destiné à être l'un des deux principaux piliers d'une maison noble, le tronc était ramené au village porté sur les épaules des hommes qui chantaient le même chant que celui qui accompagnait l'arrivée au village de têtes coupées à la guerre.

33 À la fin de la reconstruction d'une maison, on ramasse tous les débris végétaux qui restent au sol, on en met un peu dans une corbeille avec du bétel et une offrande de nourriture, là encore après avoir immolé une chèvre. Il s'agit de nettoyer la maison mais aussi de s'excuser d'avoir tué des enfants des mères de toutes les plantes qui ont été utilisées dans la construction. La corbeille est déposée dans une fourche d'arbre vers le couchant pour que les traces du forfait disparaissent à l'horizon et qu'en même temps l'offrande de nourriture soit transportée sur les cornes de la chèvre dans le monde d'En haut.

34 L'obligation de prendre soin de l'âme des arbres et d'effectuer des offrandes compensatrices destinées à leur mère mythique et la nécessité de tuer pour cela une chèvre explique que sur le territoire d'Abis on ne coupait des arbres que si c'était vraiment nécessaire.

On faisait en sorte que les habitations du tas construites sur pilotis avec leur toit couvert d'Imperata descendant jusqu'au sol (Photographie 3), aient rarement besoin d'être reconstruites; pour cela on refaisait périodiquement la toiture, les bottes d'Imperata devant se superposer en plusieurs couches afin que l'intérieur de la maison, et donc toutes ses structures, soient à l'abri de la pluie et ne puissent se détériorer (Photographie 4). 
Photographie 3 : Maisons traditionnelles au tAS d'Abis

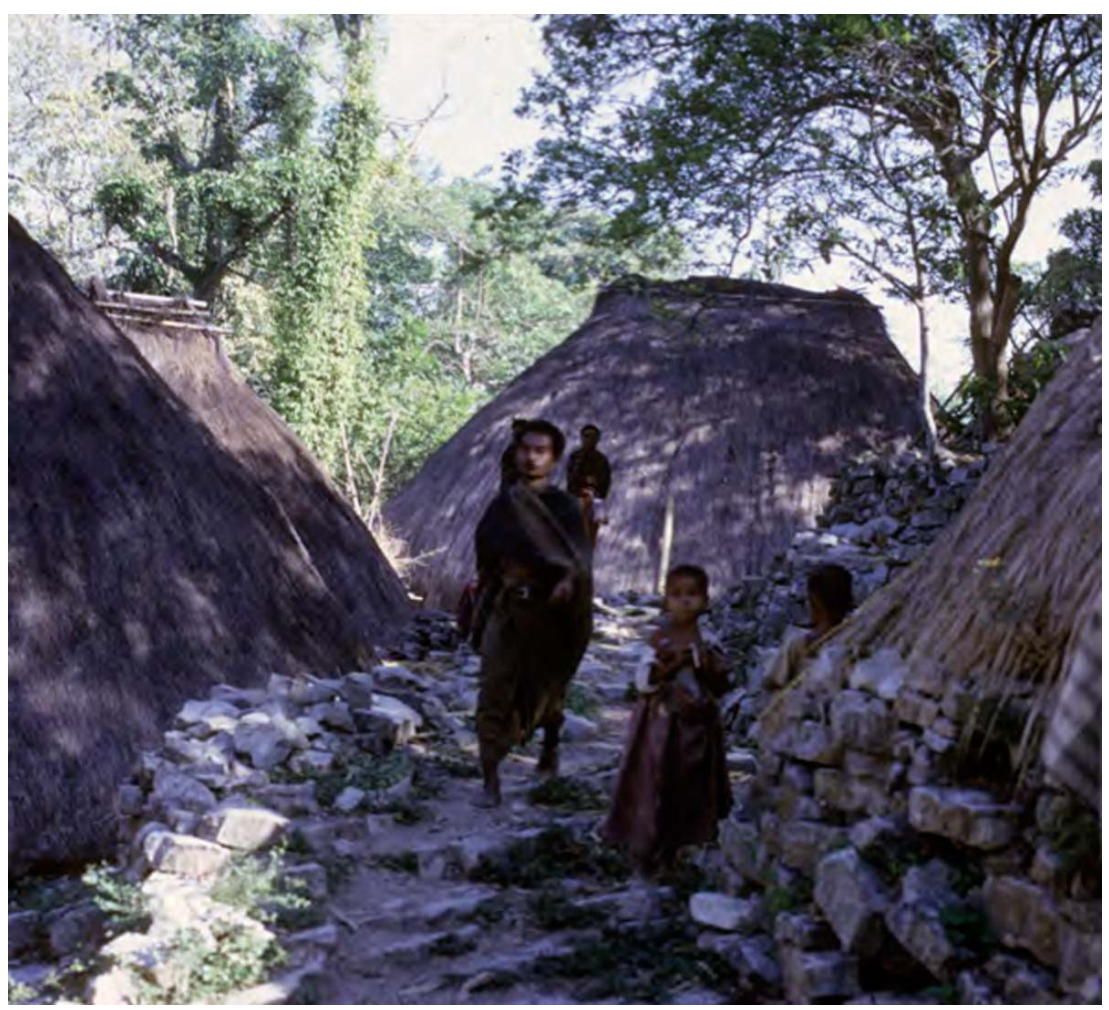

(C) C. Friedberg

Photographie 4 : Réfection du toit d'une maison traditionnelle

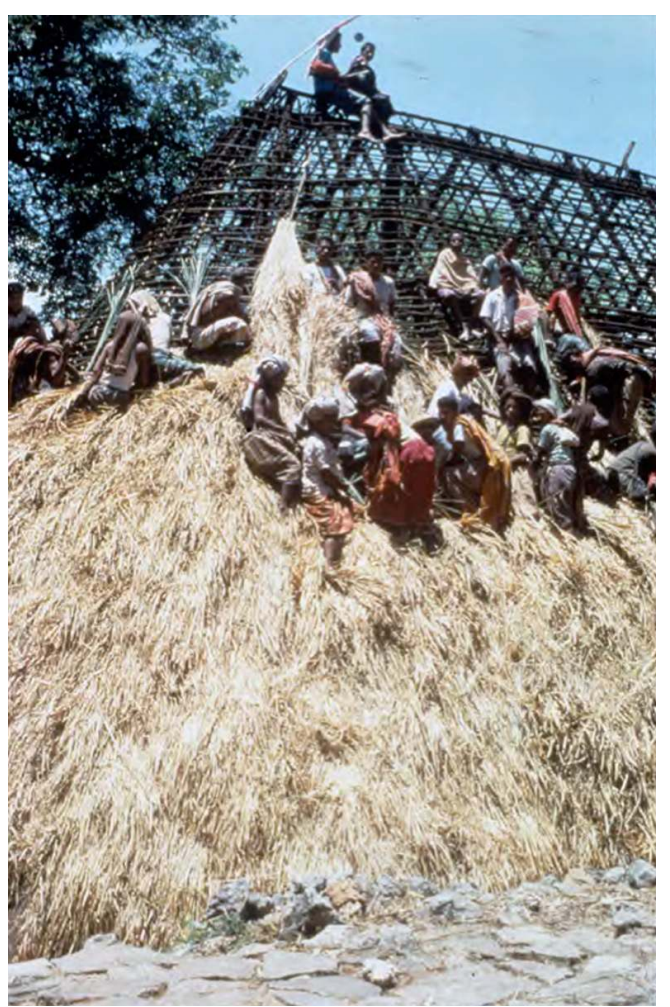

(c) C. Friedberg 

pour tâche de vérifier que les règles de gestion du territoire sont respectées, en particulier qu'aucun animal domestique n'entre dans le matas momen sous peine d'être abattu; qu'aucun prélèvement n'a été effectué sur les productions frappées d'interdit jusqu'à ce que le rituel de levée (sau) de cet interdit ne soit effectué. Il s'agit, des mangues, des noix de bancoulier, des fruits des différents palmiers (cocotiers et aréquiers). Ces levées d'interdit étaient souvent regroupées avec celle du maïs tardif, celui que l'on conserve comme nourriture de base jusqu'à l'année suivante. Pour ce maïs le rituel était d'abord collectif pour l'ensemble du village, puis pour chaque maison en 
particulier. À ces occasions, les ancêtres et défunts plus ou moins récents étaient conviés et on leur demandait aide et assistance pour que la récolte soit bonne.

Il était également nécessaire de demander l'autorisation du Kapitan pour prélever les éléments nécessaires à la construction d'une maison, non seulement des arbres mais aussi de bambous, des tiges de rotin (Daemonorops sp.), des feuilles de palmier à sucre (Arenga pinnata) pour lier les bottes d'Imperata et des fibres noires de l'infrabase des pétioles de ce même palmier utilisées pour couvrir les faîtières.

Le tas d'Abis était situé à la limite entre le matas momen et bula. Du côté de bula il y avait une barrière destinée à empêcher les porcs qui était libres de leurs mouvements d'entrer dans le village mais cela permettait aux femmes d'appeler les leurs pour les nourrir tous les soirs.

Le bosok, autel matérialisant la vitalité des habitants, était du côté du matas momen et juste derrière, l'abritant de leur ombre se trouvaient les arbres de la forêt interdite de coupe qui s'étendait jusqu'en bas de la colline (Photographie 5). En effet pour protéger leur vitalité il faut que les pana getel mone goron des villageois soient préservées de la chaleur. D'ailleurs dans le tas même, il y avait plusieurs grands arbres et des arbres fruitiers autour des maisons maintenant une certaine fraîcheur même au plus fort de la saison sèche. Pour les Bunaq la vitalité et la fertilité sont associées au froid tandis que le chaud est associé au danger et à la guerre.

Photographie 5 : Des hommes prêts à effectuer un rituel au воsok. Derrière eux, les arbres de la forêt interdite de coupe

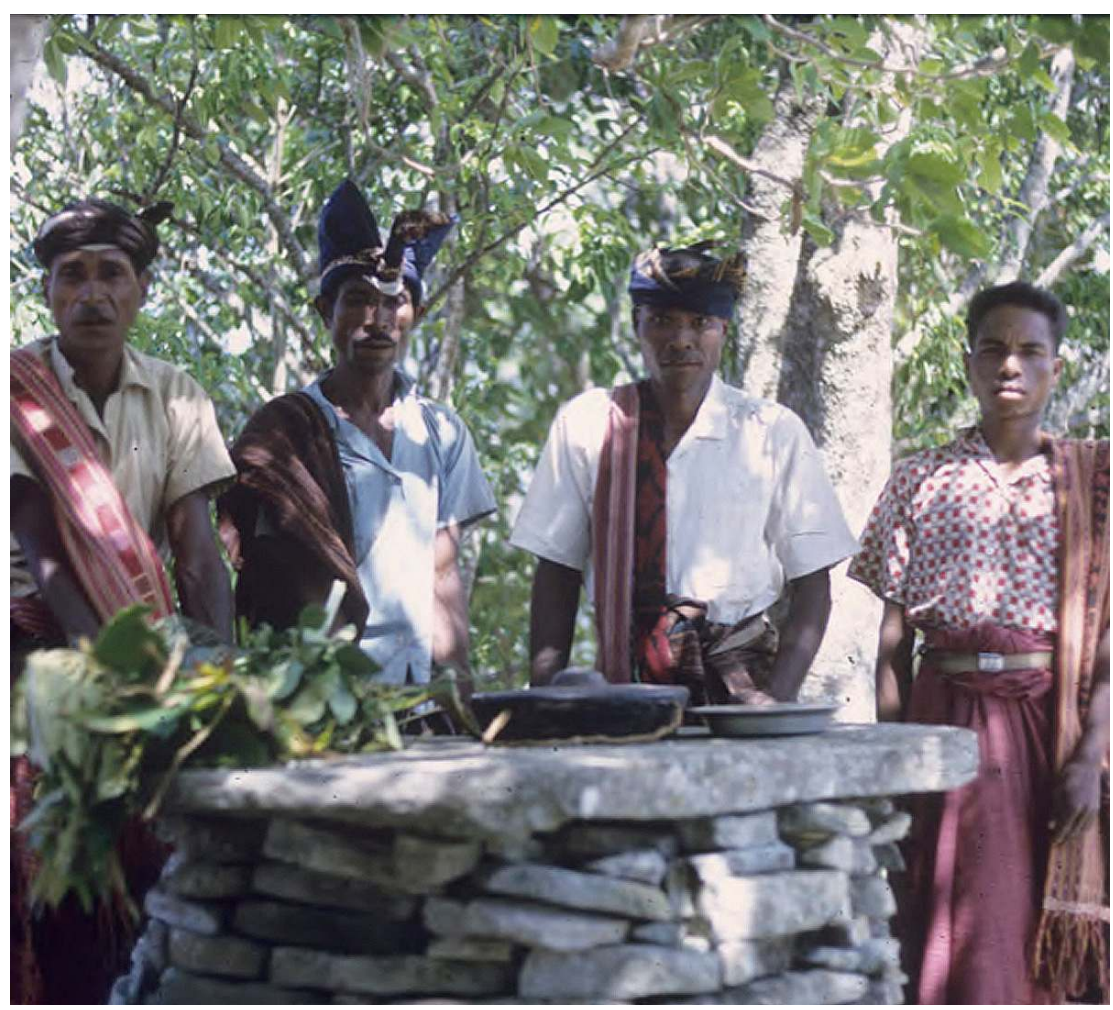

(C) C. Friedberg

Comme exemple de gestion de lieu protégé autour d'une source je prendrai celui d'un bosquet particulièrement luxuriant avec de grands arbres autour d'un point d'eau où se trouve un autel appartenant à cette même maison héritière du chef féminin autochtone ${ }^{7}$. 
L'eau de la source s'échappe dans un canal autour duquel ont été plantés des aréquiers et des cocotiers. On y trouve aussi des palmiers à sucre, des pandanus et des rotins.

C'est un lieu qui joue un rôle important pour l'ensemble de la population d'Abis à la fin de la saison des pluies. En effet le kapitan et les mak leqat viennent y chercher des « remèdes » destinés à faire venir le soleil afin que les récoltes mûrissent. Ces « remèdes " sont constitués d'écorces sèches de cocotiers et d'aréquiers et de racines de pandanus qui, bien que trempant dans l'eau, sont jaune paille et sont ainsi susceptibles d'attirer la sécheresse que l'on veut voir s'installer. Ces « remèdes » seront distribués au responsable des objets sacrés de chaque maison qui iront les déposer sur leur autel de champ au cours d'un rituel au cours duquel on installe symboliquement le soleil. Ce dernier est représenté par une palme de cocotier disposée en couronne sur un bambou.

Pendant que le kapitan et ses aides ramassent les écorces, les responsables du rituel de leur maison préparent des offrandes qui seront disposées sur l'autel qui se trouve près du point d'eau. Il est constitué par des pierres plates au-dessus desquelles se trouve une effigie d'ancêtre en pierre également (Photographie 6).

Photographie 6 : Au milieu de la végétation luxuriante du bosquet protégé, rituel sur l'autel avec effigie d'ancêtre en pierre

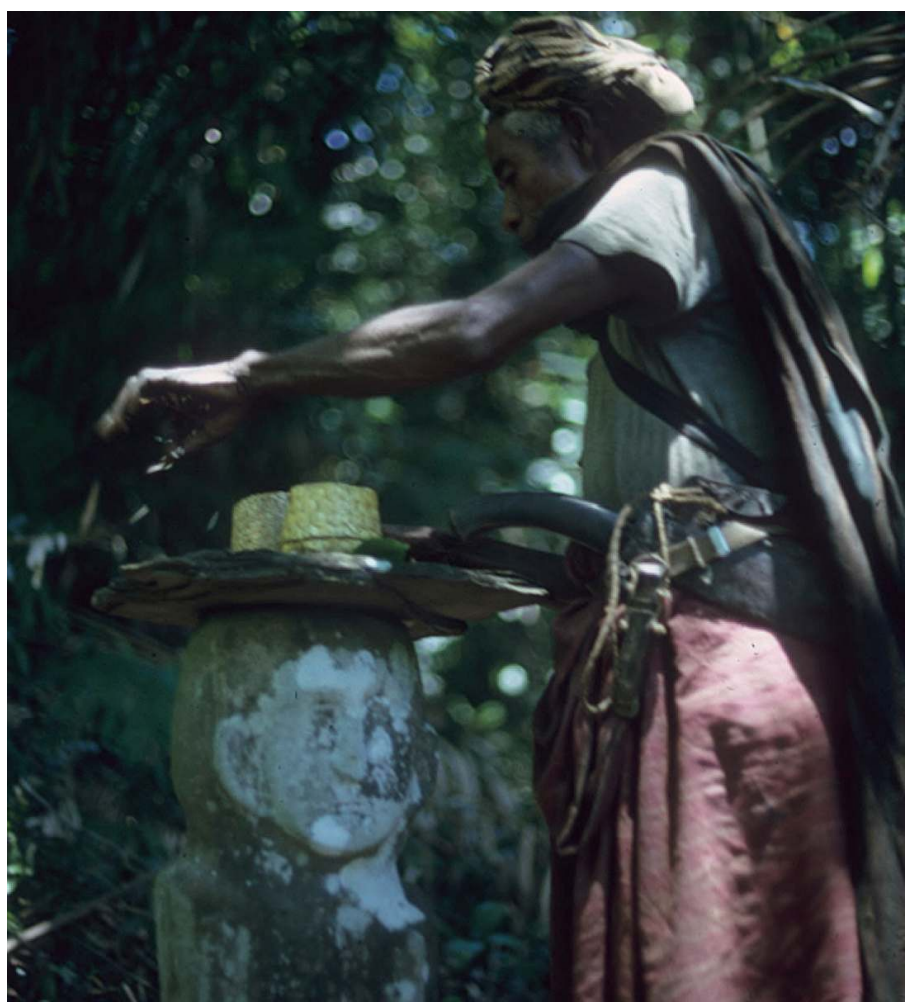

(C) C. Friedberg

51 Avant que les offrandes ne soient disposées sur l'autel et n'aient été présentées aux êtres de l'au-delà en relation avec ce lieu et après en avoir avisé le kapitan, ceux qui ont besoin de bambous, de rotin, de palmes de palmier à sucre ou des fibres de l'infrabase des pétioles de ce même palmier, peuvent se servir sans toutefois tuer les plantes ou compromettre leur capacité de reproduction. Ce sera la seule fois de l'année. En dehors de cette occasion rien ne pourra être prélevé de la végétation qui pousse sur ce lieu. 
Cet exemple permet de s'interroger sur la signification de la protection dont la végétation de ce lieu est l'objet. Est-ce parce que s'y trouve un autel ou y a-t-on mis cet autel parce que le lieu était protégé ?

La même question peut se poser pour les autres sources permanentes qui se trouvent sur le territoire d'Abis et appartiennent à une maison particulière. On y trouve un autel formé de pierres plates et parfois une effigie d'ancêtre et une végétation abondante interdite de coupe en dehors de prélèvements limités quand on y effectue un rituel. On peut se demander qui protège et qui est protégé.

Cependant on ne peut que constater la richesse très relative de la biodiversité des espèces arborées sur le territoire d'Abis. En raison des conditions climatiques et édaphiques, il s'agit d'un nombre limité d'espèces semi-décidues et de quelques espèces sempervirentes. De toute façon, la flore de Timor ne peut se comparer à celles des îles de l'archipel indonésien n'ayant pas de saison sèche marquée (Friedberg et al. 2004).

\section{Les interdits de consommation et d'utilisation}

Il me reste à aborder un aspect des pratiques pouvant entraîner la protection de certaines espèces, celui des interdits de consommation ou d'utilisation.

C'est un domaine sur lequel j'ai eu beaucoup de mal à enquêter dans la mesure où ces interdits m'ont été présentés comme propres à chaque maison et que dans certains cas même les membres de ces maisons ne semblaient pas être au courant et pas très sûrs de qui devait les observer : tous les membres de la maison, ou seulement les gardiens des objets sacrés. En outre il s'est révélé que si certains interdits paraissaient suivre des relations de filiation propre à la descendance d'un ancêtre particulier, d'autres étaient partagés par une grande partie de la population d'Abis.

57 Ces derniers semblaient liés à la présence de fragments de la plante dans le kaluk de guerre, c'est-à-dire les « remèdes » gardés précieusement dans un petit sac et censés avoir des vertus protectrices dans les combats, ou de guérison en cas de blessure. La guerre n'étant plus pratiquée depuis longtemps lors de mes enquêtes, il est normal que le souvenir des interdits liés soit estompé.

Quant aux interdits de descendance ils étaient associés à des récits mythiques d'épisodes vécus par un ancêtre dans lequel la plante a joué un rôle.

59 Ce sont les interdits d'utilisation de certaines espèces comme bois de chauffage qui pouvaient avoir le plus d'influence pour la conservation de ces espèces. Mais peu d'espèces sont concernées à Abis: Mallotus philippensis, dont il existe plusieurs exemplaires autour du bosok, de toutes façons interdits de coupe ; Cordia sp. et Bridelia ovata, toutes trois plantes de kaluk communes à tout le village. Si on les brûle on contracte des maladies de peau que l'on peut mettre en relation avec les excroissances que l'on trouve sur Bridelia.

Pour l'interdiction d'utiliser comme combustible Alstonia scholaris qui s'est transmis à trois maisons d'Abis, l'explication qui m'a été donnée est qu'un ancêtre transportant une marmite d'eau de mer a accroché celle-ci à une branche de cet arbre pour la déposer dans sa maison. 


\section{Conclusion}

61 Nous avons pu constater combien le respect que les Bunaq d'Abis ont pour les végétaux jouant un rôle dans leur vie, tient à un sentiment d'appartenance à un même univers, régi par les mêmes règles. Ces végétaux ont comme les humains des ancêtres dans le monde d'En haut et sont, là où ils vivent, dépendants des maitres du sol, à Abis, les esprits des Melus, habitants autochtones du territoire. Les végétaux ont une âme comme les humains, tandis que ces derniers ont, comme les végétaux, des racines et des feuilles garantes de leur vitalité.

Le comportement des humains vis-à-vis des végétaux répond à une volonté de préservation de la fertilité et des capacités de production de leur territoire, en particulier pour ce qui concerne la gestion des terres de culture, le matas momen, et d'une façon générale à un souci de se protéger des représailles des êtres de l'au-delà qui sont les véritables propriétaires du territoire et de ce qui y pousse, les mères et pères des végétaux et les maîtres du sol.

63 Ce n'est donc pas par hasard que soient dévolues à la maison héritière de l'ancien chef féminin des autochtones, les fonctions liées à la responsabilité vis-à-vis de la couverture végétale du territoire.

Dans la gestion de la végétation, autour des sources on est dans un processus de réciprocité entre les autels, lieux de communication avec les ancêtres, et la végétation qui les maintient dans la fraîcheur nécessaire à la vie de leurs descendants. Il en était de même pour le bois protégé autour d'Abis et de son bosok représentant les racines et les feuilles de ses habitants.

Si on se réfère aux ontologies qui d'après Descola (2005) caractérisent les relations que les humains entretiennent avec les autres êtres vivants, on peut constater que les pratiques des habitants d'Abis relèvent de deux ontologies, l'animisme et l'analogisme. L'analogisme est nettement mis en œuvre à travers la performativité des rituels. On peut également se demander si certains interdits d'utilisation ou de consommation transmis par filiation ne relèveraient pas de l'ontologie totémique (Friedberg 2007).

\section{Épilogue}

Dans les premier mois des combats provoqués par l'invasion de la partie portugaise de l'île par l'armée indonésienne, les Bunaq ont fui cette zone de Lamaknen. Le tas d'Abis a brûlé et le feu s'est propagé au bois protégé. La colline, n'étant plus protégée de l'érosion, s'est en partie écroulée.

67 Comme j'ai pu le constater en 1997, certains des habitants d'Abis étaient revenus sur leur territoire et s'étaient installés dans des maisons individuelles le long de la route, construite sur le bula qui va jusqu'à la frontière avec Timor Leste.

Ces maisons étaient entourées de jardins contenant de nombreux bananiers et papayers, l'ensemble étant protégé par des grands arbres. Sont-ils des reliques de la végétation en place épargnées lors de la construction des habitations, ou ont-ils été plantés ? L'essentiel est qu'ils remplissent leur fonction : préserver les humains du chaud. 


\section{BIBLIOGRAPHIE}

Descola P. 2005 - Par delà nature et culture. Paris, Gallimard, 640 p. (Bibliothèque des Sciences humaines).

Donohue M. \& Denham T. 2010 - Farming and Language in Island Southeast Asia. Reframing Austronesian History. Current Anrhropology 51 (2) : 223-256.

Friedberg C. 1977 - La femme et le féminin chez lesBunaq du centre de Timor. Archipel 13 : 37-52.

Friedberg C. 1982 - Muk Gubul Nor, "La chevelure de la terre". Les Bunaq et les plantes. Thèse de Doctorat d'État es Lettre et Sciences Humaines, Paris V.

Friedberg C. 1990 - Le savoir botanique des Bunaq : percevoir et classer dans le Haut Lamaknen, Timor, Indonésie. Paris, Muséum national d'histoire naturelle, 304 p. (Mémoires du Muséum national d'histoire naturelle. Série B, Botanique ; 32).

Friedberg C. 2007 - Par-delà le visible. Natures Sciences Sociétés 15 (2) :167-176.

Fiedberg C., Purwanto Y., Suharno D.M., Waluyo E.B. 2004 - Lieux de culte, végétation, ancêtres, en Indonésie de l'Est : qui protège et qui est protégé ? Journal of Tropical Ethnobiology 1 (2) : 12-29.

O'Connor S. 2007 - New evidence from East Timor contributes to our understanding of earliest modern human colonisation east of the Sunda Shelf. Antiquity 81 : 523-535.

Oliveira Nuno Vasco da Siva Miranda de 2008 - Food Production and the Agricultural Transition in East Timor. PhD of the Australian National University, Camberra.

Schapper A. 2011 - Crossing the border: historical and linguistic devides among the Bunaq in central Timor. Wacana, Journal of the Humanities of Indonesia 13 (1) : 29-49.

\section{NOTES}

1. Lamaknen est situé dans l'île de Timor, dans le district Belu de la Province de Nusa Tenggara Timur en Indonésie.

2. On peut trouver les données que j'ai rassemblées lors de mes missions de recherche sur le terrain dans mon mémoire de thèse d'État (Friedberg 1982).

3. L'attribution d'une polarité féminine (pana) ou masculine (mone), n'a pas toujours de relation avec le sexe des êtres auxquels elle s'applique. Par exemple, pour les plantes, le qualificatif pana est ajouté au terme d'appellation pour distinguer entre deux formes proches, celle qui a les feuilles les plus grandes ou pour un type d'igname celui qui a la peau la plus fine ; c'est-à-dire que d'une façon générale, c'est ce qui est le plus valorisé qui est qualifié de «féminin » (Friedberg, 1990). Le terme pana est aussi utilisé comme substantif et désigne alors une femme (Friedberg, 1977).

4. Le santal a constitué pendant longtemps la richesse de Timor et alimenté un commerce interinsulaire. À l'époque de mes recherches il n'en restait plus beaucoup sur le territoire d'Abis.

5. Les six photographies présentées datent du début des années 1970.

6. Les noms scientifiques des plantes citées dans cet article sont repris de Friedberg 1990 sans avoir été actualisés.

7. On trouvera des données plus complètes sur ce bosquet sacré dans Friedberg et al. 2004. 


\section{RÉSUMÉS}

Les pratiques ayant pour résultat la conservation de certains éléments de l'environnement peuvent avoir des motivations multiples, souvent éloignées de celles des organismes scientifiques internationaux de conservation de la nature.

Je me propose de décrire les pratiques que j'ai pu observer dans les années 70 chez une population du centre de Timor dans la partie indonésienne de l'île à Abis un village de langue bunaq.

Ces pratiques sont de deux types:

celles qui concernent l'organisation du territoire partagé entre terres de culture et terres de pâture et les règles associées, en particulier la protection des plantes utiles lors des brûlis de préparation des champs.

Celles qui concernent la végétation interdite de coupe dont le statut est plus ambigu. Est-ce la végétation qui est protégée ou cette végétation est-elle préservée pour protéger des autels ayant une fonction dans la société?

There are various reasons to explain the practices that result in preserving certain parts of the environment. But these reasons are often very different from those brought up by the international scientific organisms aiming to preserve the nature.

In this article, I will describe the practices I have had the opportunity to observe in the 1970's within a central Timor population, based in the Indonesian part of the island.

This population lives in Abis, a bunag-speaking village.

The practices are twofold:

Those concerning the organization of the territory - divided between the farming land and the grazing land - and their associated rules; especially aiming to protect the useful plants in burnt land.

Those concerning the vegetation that is forbidden to trimming and whose status is much more ambiguous. Is the vegetation protected for itself or in view to protect the altars, which have a true meaning in society?

\section{INDEX}

Keywords : Timor, Bunaq, agrobiodiversity, protection, reciprocity, ancestors, animism, analogism

Index géographique : Timor, Indonésie (NTT)

Population Bunaq

Mots-clés : végétaux, agrobiodiversité, protection, réciprocité, ancêtres, animisme, analogisme 


\section{AUTEUR}

\section{CLAUDINE FRIEDBERG}

Anthropologue, Département Hommes, Natures, Sociétés, Muséum national d'histoire naturelle, Bâtiment 135, 57 rue Cuvier, 75231 Paris cedex 05, France

friedbg@mnhn.fr 